\title{
Imaging the LHC beams with silicon and scintillating fibre vertex detectors
}

\author{
M. Rihl ${ }^{\mathrm{a}, \mathrm{b}, *}$, On behalf of the LHCb and BGV Collaborations \\ ${ }^{a}$ CERN, Geneva, Switzerland \\ ${ }^{\mathrm{b}}$ Vienna University of Technology, Austria
}

\section{A R T I C L E I N F O}

\section{Article history:}

Received 25 March 2016

Accepted 15 April 2016

Available online 16 April 2016

Keywords:

BGV

Beam gas interaction

VELO

LHCb

CERN

\begin{abstract}
A B S T R A C T
The LHCb Vertex Locator (VELO) is used to reconstruct beam-gas interaction vertices which allows one to obtain precise profiles of the LHC beams. In LHCb, this information is combined with the profile of the reconstructed beam-beam collisions and with the LHC beam currents to perform precise measurements of the luminosity. This beam-gas imaging (BGI) method also allows one to study the transverse beam shapes, beam positions and angles in real time. Therefore, a demonstrator beam-gas vertex detector (BGV) based on scintillating fibre modules has been built and installed in LHC Ring 2 at point 4.

(C) 2016 The Authors. Published by Elsevier B.V. This is an open access article under the CC BY license
\end{abstract} (http://creativecommons.org/licenses/by/4.0/).

\section{Introduction}

Beam imaging is an important tool in accelerator based physics experiments. Measured parameters for single beam diagnostics are among others the position of the colliding beams in the accelerator, the transverse and longitudinal beam profile as well as the beam intensity. Assuming Gaussian bunches and no dispersion, the transverse beam size $\sigma$ is related to the beam emittance $\epsilon$ and the local beam optics function $\beta$ (for a deeper insight into the accelerator parameters see [1]):

$\sigma^{2}=\epsilon \beta$.

For collider experiments, luminosity is the parameter that links the beam properties of single beams with physics rates. Hereby, the luminosity $L$ is the quantity that relates the cross section $\sigma_{\text {proc }}$ of a physics process to the interaction rate $R$. For a colliding bunch pair, one has:

$R=L \sigma_{\text {proc }}, \quad L=f_{\text {rev }} N_{1} N_{2} \Omega$,

where $N_{i}$ is the bunch population of beam $i(i=1,2)$ and $f_{\text {rev }}$ is the revolution frequency of the beam in the accelerator. The beam overlap integral $\Omega=2 c \int \rho_{1}(x, y, z, t) \rho_{2}(x, y, z, t) d x d y d z d t$ embodies the passage of the two bunches with spatial particle density distributions $\rho_{1}(x, y, z, t)$ and $\rho_{2}(x, y, z, t)$ across each other.

There are different methods to measure luminosity. In the LHCb experiment at CERN, the van der Meer method (VdM) and the beam-gas imaging method (BGI) are used.

\footnotetext{
* Correspondence address: CERN, Geneva, Switzerland.
}

The LHCb experiment [2] at the CERN Large Hadron Collider (LHC) is a forward detector that was designed to study particles containing $b$ or $c$ quarks. It consists of several sub-systems, like the vertex locator (VELO), ring imaging Cherenkov detectors, a dipole magnet, further tracking detectors, a calorimeter system and a muon identification system. The LHCb VELO is an important instrument in the measurement of the luminosity with both VdM and BGI methods. It consists of two halves, each made up of 42 silicon strip sensors (21 $r$-measuring and $21 \phi$-measuring) installed inside the LHC vacuum which can be moved to a distance of $8.2 \mathrm{~mm}$ from the LHC beams. The VELO allows us to reconstruct vertices from beam-beam interactions as well as beam-gas interactions, see Fig. 1. Its resolution plays a crucial role in the BGI method.

\section{Results of the BGI method}

The BGI method [3], which premiered at LHCb, takes advantage of beam-gas interactions inside the VELO into which (noble) gas is injected. The bunches of the two beams collide with the gas and each other. The reconstructed vertices give direct information about the $\rho_{i}$ 's, crossing angle, offset, etc. These results are used for luminosity calibrations. Typical beam-gas rates per LHC bunch of $10^{11}$ protons are between $50 \mathrm{~Hz}$ and $100 \mathrm{~Hz}$. In June 2015, preliminary results were obtained for proton-proton collisions at $\sqrt{s}=13 \mathrm{TeV}$ which gave an absolute reference cross section of $63.40 \pm 2.47 \mathrm{mb}( \pm 3.9 \%)$.

In addition to providing another method of luminosity calibration the BGI method also gives an image of the structure of the 
LHCb preliminary

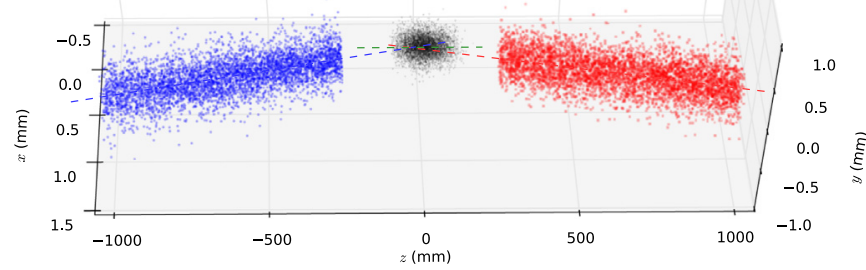

Fig. 1. Reconstructed interaction vertices, blue dots show interactions of beam 1 with gas, red dots the interactions between beam 2 and gas while black dots in the middle show the luminous region of beam 1 and beam 2. (For interpretation of the references to colour in this figure caption, the reader is referred to the web version of this paper.) bunches inside the LHC and allows tests of the beam shape description [3]. In Figs. 2 and 3, the beam shape fits of both beams are compared with the beam shape description and the deviation of the data to the model is plotted. This gives an insight into the actual bunch shape compared to the assumed bunch shape.

\section{Limitations of the VELO BGI method}

The LHCb BGI method provides precise measurements of the luminosity and beam profiles when the VELO is closed. However, its performance is degraded when the LHC is not in "STABLE BEAMS" and the VELO detectors are in retracted position. A closed position of the two VELO halves during injection and energy ramping is prohibited by design because of possible beam orbit excursions and other detector protection issues.

\section{$\mathrm{LHCb} \quad$ Beam 1}

data

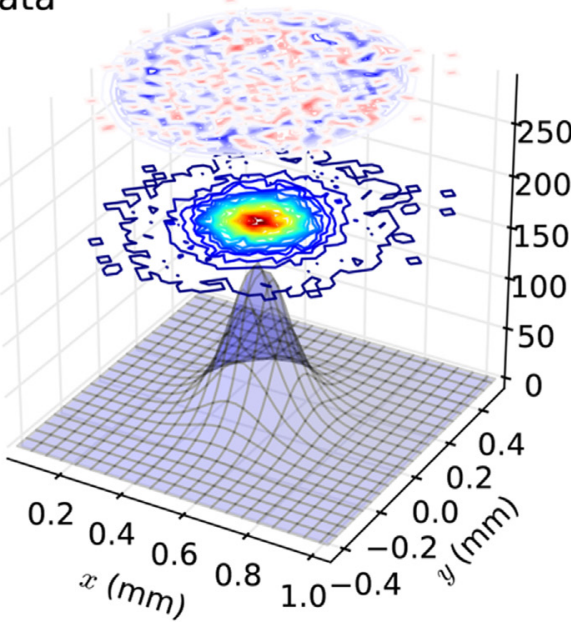

Beam 2

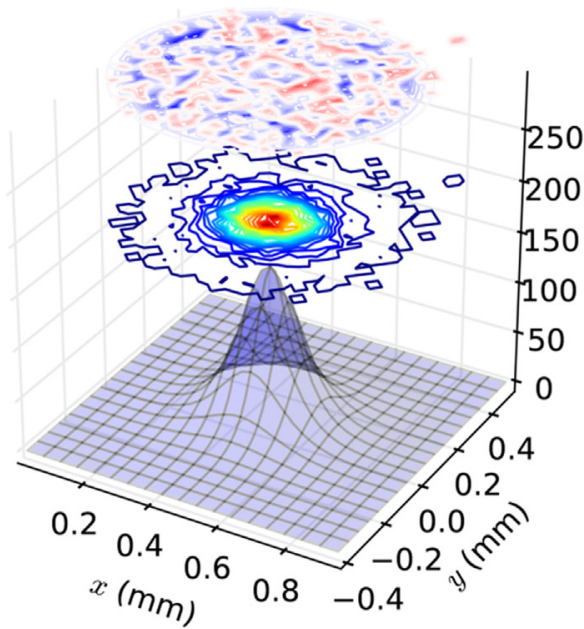

Beam-beam

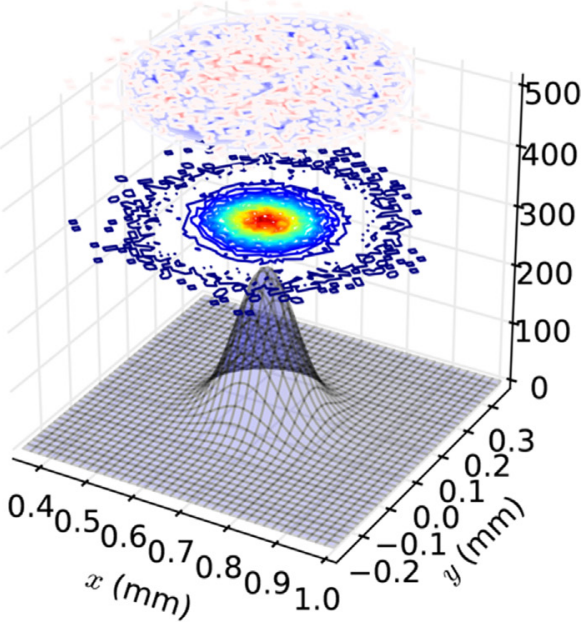

Fig. 2. Comparison of fit of measured bunch transverse distribution and model for a selected $z$-slice of beam 1 (left), beam 2 (middle) and the luminous region (right).

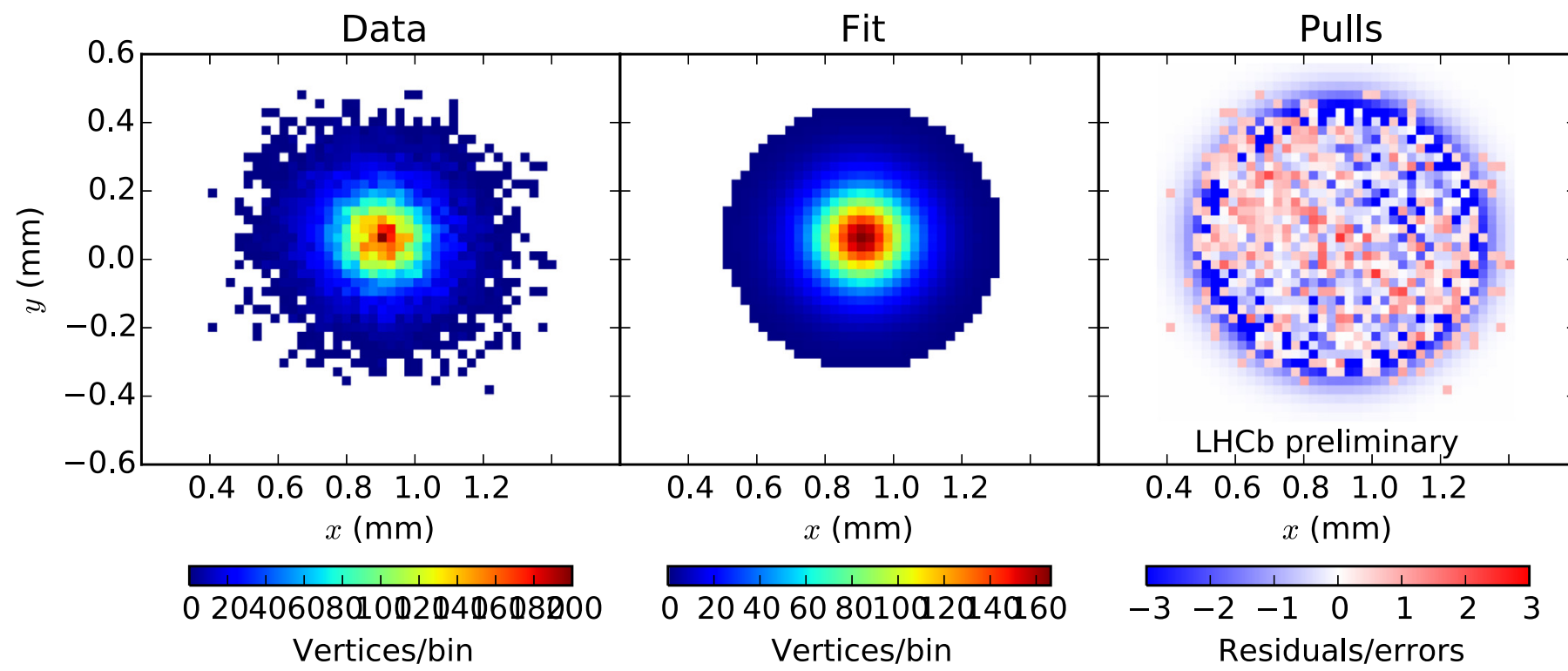

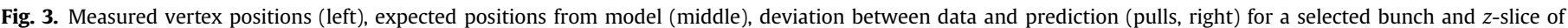
beam 1. 


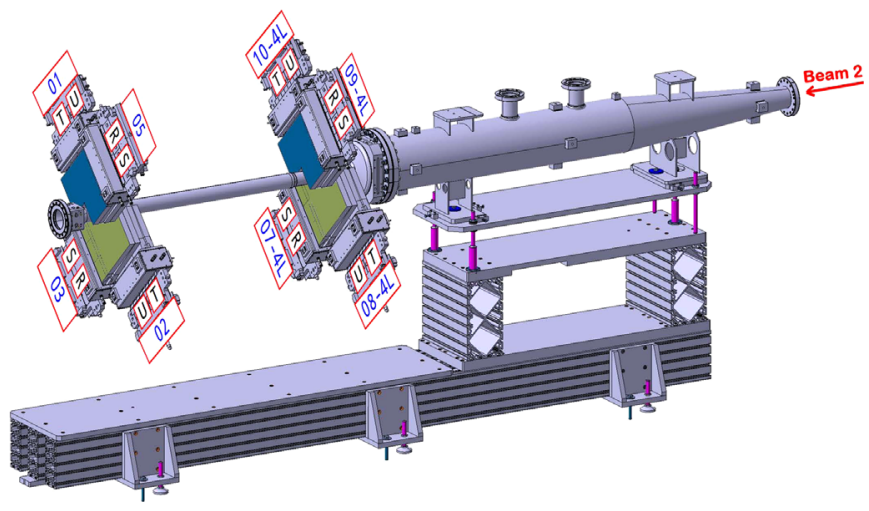

Fig. 4. Sketch of the BGV at the LHC point 4.

\section{The beam gas vertex detector - BGV}

In order to measure the transverse beam profile and thus the beam width during all states and energies of the LHC, a dedicated device, a demonstrator beam-gas vertexing detector (BGV) [4], was designed, developed and recently installed in point 4 on LHC Ring 2.

At the BGV, similar to the BGI method, a noble gas (neon or argon) is injected into a vacuum chamber. The interactions between the beam and the gas are detected by 8 scintillating fibre modules (see Fig. 4), each containing two fibre mats, where one mat is rotated by $2^{\circ}$ to the other one. The scintillating fibre mats and photodetectors were developed in close collaboration with the Scintillating Fibre (SciFi) Tracker project of the LHCb upgrade [5]. The scintillating fibres have a diameter of $250 \mu \mathrm{m}$ and a length of $240-340 \mathrm{~mm}$. For dimensions and the shape of the detector modules, see Fig. 5.

Simulations show that the BGV vertex resolution is dominated by multiple scattering. In order to reduce the material close to the interaction point, modules with 4 layers of scintillating fibres were installed in the station close to the vacuum chamber while 5-layer modules were installed at the station far from the vacuum chamber. The expected number of photons per MIP is around 20 for the 4-layer modules and 25 for the 5-layer modules.

The fibres are read out by silicon photo-multipliers (SiPMs) and the light yield is increased by using a mirror on the other end of the fibres. The modules include a cooling and a dry air system to cool down the SiPMs to $-40{ }^{\circ} \mathrm{C}$. The SiPMs are read out with radiation hard, analogue $\mathrm{LHCb}$ Beetle front-end chips that are also used in the VELO. Since the BGV's SiPMs have a larger output current than the silicon strip sensors of the VELO, special frontend boards were developed to attenuate the SiPM signals to match the Beetles' characteristics. Each SiPM (128 channels) is connected to one Beetle through an attenuator circuit. In total 16 Beetles and SiPMs are used for one module. The Beetle signal gets amplified in a VELO repeater board (one per module) to drive the signal over $60 \mathrm{~m}$ to the TELL1 readout boards. These TELL1 boards, as well as all other non-radiation hard electronics are installed in the service tunnel parallel to the LHC tunnel, $60 \mathrm{~m}$ from the BGV, see Fig. 6 . The TELL1s (one per module) convert the analogue signal into digital data, apply zero-suppression (clusterisation) and send multi-event packets to a computing farm. The BGV computing farm consists of a server rack with a blade enclosure which is currently filled with three blade servers. The blade enclosure can be filled with up to 16 blade servers to process the data in real time. For commissioning the BGV system, data are analysed offline; after commissioning, the track and vertex reconstruction will be performed on the fly in the BGV CPU farm with the aim to produce a real-time image of the LHC beam and individual bunches.

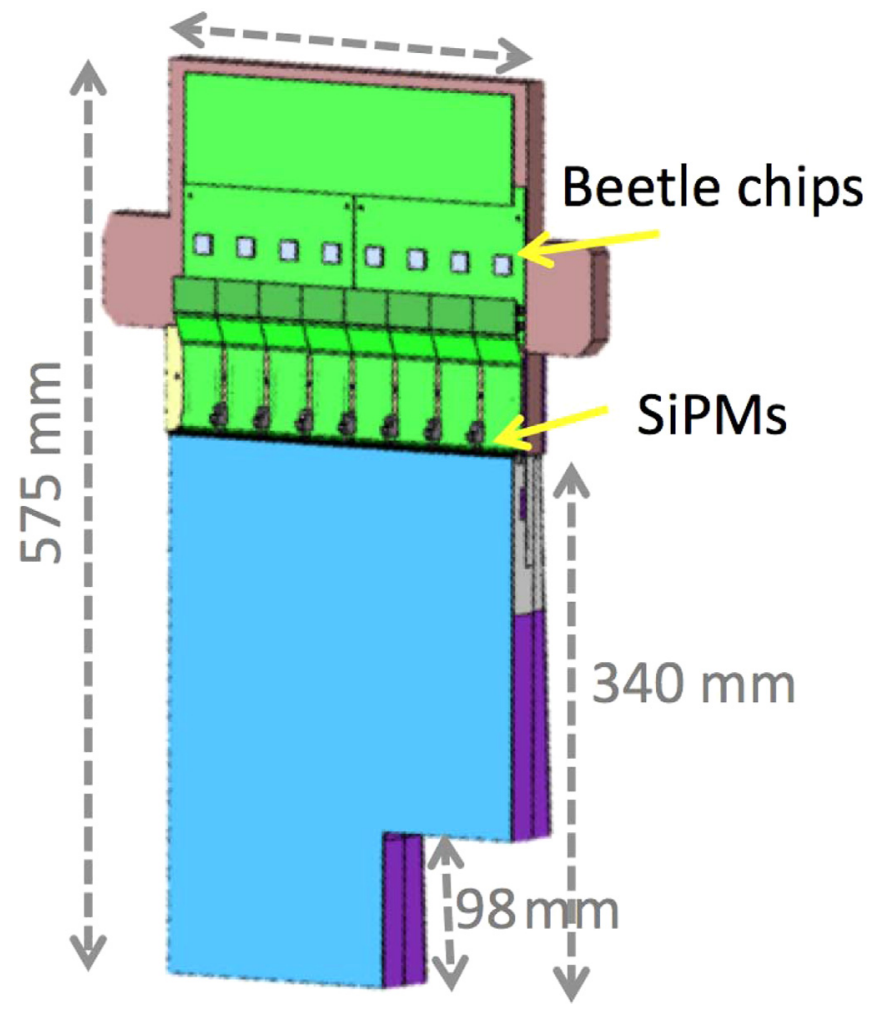

Fig. 5. Sketch of a BGV module with scintillating fibre mats in cyan and front-end electronics in green. (For interpretation of the references to colour in this figure caption, the reader is referred to the web version of this paper.)

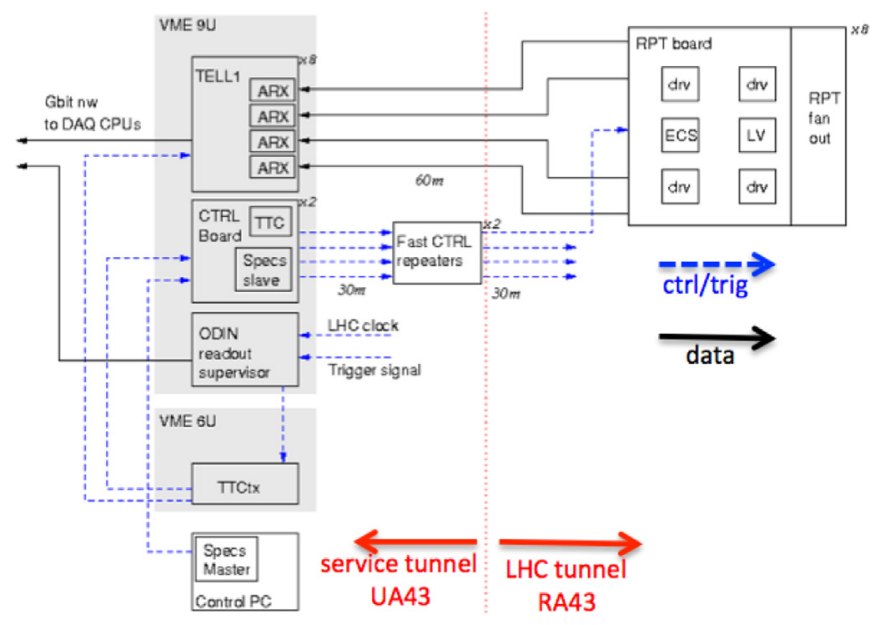

Fig. 6. Sketch of the BGV readout and control system.

\section{Radiation impacts}

Since the detector modules are installed as close as possible to the beam pipe, radiation does have an impact on the modules. The distance between the scintillating fibres and the beam is approximately $26 \mathrm{~mm}$ while the distance between the SiPMs and the beam is about $268 \mathrm{~mm}$ or bigger. Similar to LHCb SciFi tracker SiPMs, the expected yearly dose for the BGV SiPMs is of the order of $10 \mathrm{~Gy}$. For the BGV, mainly the SiPMs will be affected by radiation while in LHCb both the fibres and SiPMs are sizeably affected by radiation. The irradiation of the SiPMs results in an increased dark current over time and potentially more noise clusters. Due to these predicted effects, a cooling system was designed to 

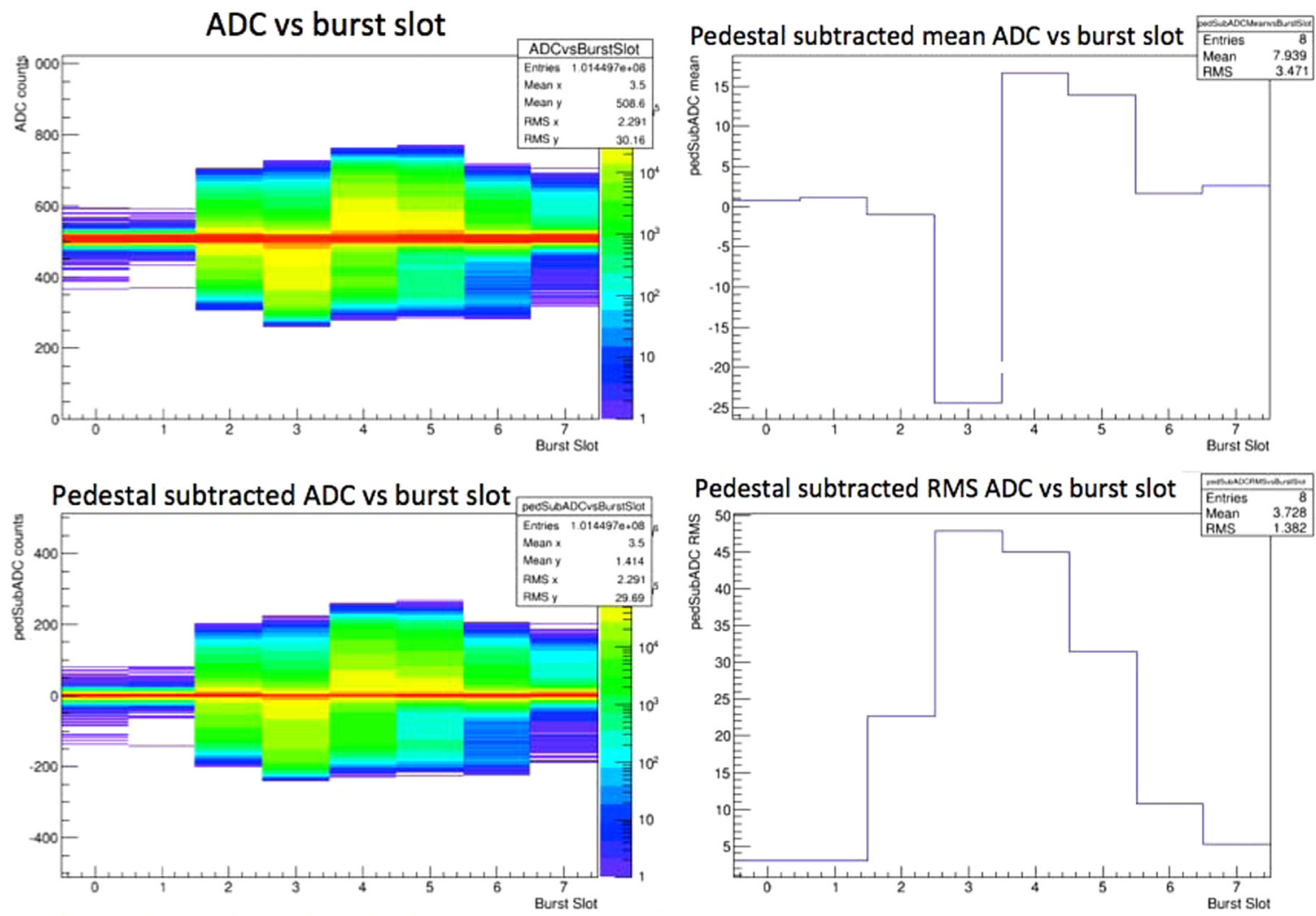

Burst slot:25ns spacing between triggers

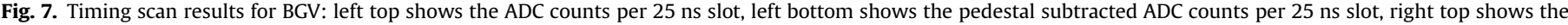
pedestal subtracted mean ADC per slot and right bottom the pedestal subtracted RMS ADC count per slot. The best delay setting is a delay that equals slot 4 .

cool down the SiPMs to temperatures as low as $-40^{\circ} \mathrm{C}$. The radiation effect on the BGV's scintillating fibre mats is negligible since the expected interaction rate (appr. $70 \mathrm{~Hz}$ per bunch) is too low to cause a substantial loss of light yield at the fibre end.

\section{Current status of the BGV}

The BGV was installed in the second half of 2015 and is currently under commissioning. In order to acquire data that can be used for track and vertex reconstruction, the time alignment of the different electronic components has to be exact. To sample at the correct 25 ns time slot, time alignment scans were performed, and an optimal trigger delay was chosen from the results of the scan, see Fig. 7. In addition to the coarse 25 ns delay, the correct fine sampling delay needs to be set.

Further ongoing work includes the development of algorithms for correcting cross talk effects (e.g. due to Beetle header bits or the attenuator circuit), for common mode subtraction and for clusterisation. Once developed and tested on raw data (non-zero suppressed data), these algorithms will be implemented in the TELL1 FPGAs and used to acquire cluster data at a higher rate. Track reconstruction and vertexing algorithms have been prepared and tested on simulation data.

\section{Summary}

The usability of the LHCb BGI method for beam imaging was demonstrated during LHC Run1 (2009-2013) and used in 2015 to rapidly obtain a luminosity calibration. These results raised interest to measure transverse beam profiles using beam gas imaging. The BGV demonstrator project was started in October 2012. This system will be able to measure the transverse bunch profile at any LHC beam intensity and throughout all phases of the accelerator cycle, thus giving insight into the beam width and emittance evolution of the beam. The development of the BGV profits from synergies with the SciFi tracker development at LHCb. The BGV detector was recently installed and is now being commissioned. First data from this device will be acquired in the 2016 LHC run.

\section{References}

[1] J. Buon, Beam phase space and emittance, CAS, CERN 91-04.

[2] The LHCb Collaboration, The LHCb detector at the LHC, J. Instrum. 3 (8) (2008) S08005. URL 〈http://stacks.iop.org/1748-0221/3/i=08/a = S08005〉.

[3] The LHCb Collaboration, Precision luminosity measurements at LHCb, J. Instrum. 9 (12) (2014) P12005, doi:http://stacks.iop.org/1748-0221/9/i=12/ $\mathrm{a}=\mathrm{P} 12005$.

[4] P. Hopchev, et al., A beam gas vertex detector for beam size measurement in the LHC, in: Proceedings of 5th International Particle Accelerator Conference (IPAC 2014), 2014, THPME175.

[5] The LHCb Collaboration, LHCb tracker upgrade technical design report, Technical Design Report LHCb 15 (CERN-LHCC-2014-001. LHCB-TDR-015). 